OPEN ACCESS

Edited by:

Yuli Huang,

Southern Medical University, China

Reviewed by:

Zhongheng Zhang,

Sir Run Run Shaw Hospital, China

Zhenggang Bai,

Nanjing University of Science and

Technology, China

*Correspondence:

Jun Lyu

lyujun2020@jnu.edu.cn

†These authors have contributed equally to this work

Specialty section: This article was submitted to

Cardiovascular Metabolism,

a section of the journal

Frontiers in Cardiovascular Medicine

Received: 13 July 2021

Accepted: 10 August 2021

Published: 10 September 2021

Citation:

Zheng S, Zhao F, Yang R, Wu W,

Liu H, Ma W, Xu F, Han D and Lyu J (2021) Using Restricted Cubic Splines to Study the Trajectory of Systolic Blood Pressure in the Prognosis of Acute Myocardial Infarction. Front. Cardiovasc. Med. 8:740580

doi: 10.3389/fcvm.2021.740580

\section{Using Restricted Cubic Splines to Study the Trajectory of Systolic Blood Pressure in the Prognosis of Acute Myocardial Infarction}

\author{
Shuai Zheng ${ }^{1,2 t}$, Fengzhi Zhao ${ }^{3 \dagger}$, Rui Yang ${ }^{4}$, Wentao Wu ${ }^{4}$, Hui Liu ${ }^{5}$, Wen $\mathrm{Ma}^{4}$, \\ Fengshuo $\mathrm{Xu}^{4}$, Didi Han ${ }^{4}$ and Jun Lyu ${ }^{1 *}$ \\ ${ }^{1}$ Department of Clinical Research, The First Affiliated Hospital of Jinan University, Guangzhou, China, ${ }^{2}$ School of Public \\ Health, Shaanxi University of Chinese Medicine, Xianyang, China, ${ }^{3}$ Intensive Care Unit, The First Affiliated Hospital of Jinan \\ University, Guangzhou, China, ${ }^{4}$ School of Public Health, Xi'an Jiaotong University Health Science Center, Xi'an, China, \\ ${ }^{5}$ School of Public Health, Lanzhou University, Lanzhou, China
}

Background: Acute myocardial infarction (AMI) is still the most serious manifestation of coronary artery disease. Systolic blood pressure (SBP) is the best predictor of blood pressure in AMI. Thus, its influence on AMl is necessary to be explored.

Methods: A total of 4,277 patients with AMI were extracted from the Medical Information Mart for Intensive Care database. Chi-square test or Student's $t$-test was used to judge differences between groups, and Cox regression was used to identify factors that affect AMl prognosis. SBP was classified as low (<90 mmHg), normal $(90-140 \mathrm{mmHg}$ ), or high (>140 mmHg), and a non-linear test was performed. Meaningful variables were incorporated into models for sensitivity analysis. Patient age was classified as low and high for subgroup analysis, and the cutoff value of the trajectory was identified. $P<0.05$ indicates statistical significance.

Results: The effect of SBP on the prognosis of patients with AMI is non-linear. The risks in models 1-3 with low SBP are 6.717, 4.910, and 3.080 times those of the models with normal SBP, respectively. The risks in models 1-3 with high SBP are 1.483, 1.637, and 2.937 times those of the models with normal SBP, respectively. The cutoff point $(95 \%$ confidence interval) of the trajectory is $114.489 \mathrm{mmHg}(111.275-117.702 \mathrm{mmHg}$, all $P$ $<0.001)$.

Conclusions: SBP has a non-linear effect on AMl prognosis. Low and high SBP show risks, and the risk of low SBP is obviously greater than that of high SBP.

Keywords: SBP, AMI, prognosis, non-linear, MIMIC

\section{INTRODUCTION}

The mortality and morbidity of acute myocardial infarction (AMI) are declining in most countries, especially in countries with higher per capita income (1-3). However, the prevalence of long-term AMI is also increasing with the aging of the world population and rapid population growth; thus, the disease burden of AMI is increasing (1). Every year, 2.4 million people die in the United States, and four million people die in Europe and North Asia; coronary artery disease (CAD) causes up to 
one-third of these deaths (4-7). AMI is the most serious manifestation of CAD and greatly increases the mortality rate of CAD (8).

The pathogenesis of acute coronary syndrome is a complex pathophysiological process accompanied by complex neuroendocrine changes (9). Neuroendocrine response after AMI results in the activation of the sympathetic nervous system and renin-angiotensin system and the release of vasopressin and atrial natriuretic peptide. The net effect of this response is vasoconstriction, cardiac stimulation, and regional flow redistribution, which may have a favorable effect in some situations and a deleterious effect in others. Therefore, blood pressure (BP) measurement can be used to reflect the overall potential performance of the cardiovascular and neuroendocrine systems after AMI (10).

As early as the 1990s, studies have shown that systolic blood pressure (SBP), diastolic blood pressure (DBP), and pulse pressure are risk factors for cardiovascular disease (11). A study in 2000 showed that the average values of SBP, DBP, and mean blood pressure (MBP) are important predictors of cardiovascular disease in young men ( $<60$ years old), and mean SBP and pulse pressure are important predictors in elderly men ( $>60$ years old) (12). In 2015, Sundström and Arima found that SBP and DBP are very important independent risk factors for cardiovascular and renal diseases (13). The various indicators of BP have played an irreplaceable warning role in the treatment and prognosis of cardiovascular diseases.

Admission SBP can be used as a predictor of rapid clinical evaluation and poor cardiovascular prognosis studies. If this indicator can be effectively used to assess the risk of adverse consequences, then a treatment plan for patients with new AMI can be quickly developed (14). Therefore, a more comprehensive and detailed understanding of the performance of SBP in the prognosis of AMI is important. The purpose of this study was to use restricted cubic splines to study the performance of SBP in patients with AMI from the Medical Information Mart for Intensive Care (MIMIC) database.

\section{MATERIALS AND METHODS}

\section{Patients and Variables}

The MIMIC [database jointly issued by the Massachusetts Institute of Technology (MIT) Computational Physiology Laboratory, Beth Israel Dikang Medical Center, and Philips Medical] is supported by the National Institutes of Health to promote the work of intensive medical research (15). MIMIC is a publicly available dataset developed by the MIT Computational Physiology Laboratory that includes unidentified patient health data related to $\sim 60,000$ intensive care unit visits. The dataset includes demographic information, vital signs, laboratory tests, drugs, and other information (16). The database has a large number of samples, comprehensive information, and long-term patient tracking; can be used for free; and provides a wealth of resources for intensive care research (17). Access to the database (Certificate Number: 38489997) was granted after the completion of the National Institutes of Health's web-based training course, "Protecting Human Research Participants." In the present study, we extracted 4,277 AMI data from the MIMIC database. All patients were diagnosed with AMI for the first time upon admission. The following variables were extracted from the information of the patients with AMI in the MIMIC database: gender, atrial fibrillation, atrial flutter (AFL), ventricular fibrillation (VF), ventricular tachycardia (VT), SBP, drug, total calcium level, chloride level, creatinine level, phosphate level, potassium level, sodium level, nitrogen level, hemoglobin count, platelet count, red blood cell width (RDW), white blood cell (WBC) count, age, respiration rate, mean heart rate, mean BP, DBP, mean glucose level, Sequential Organ Failure

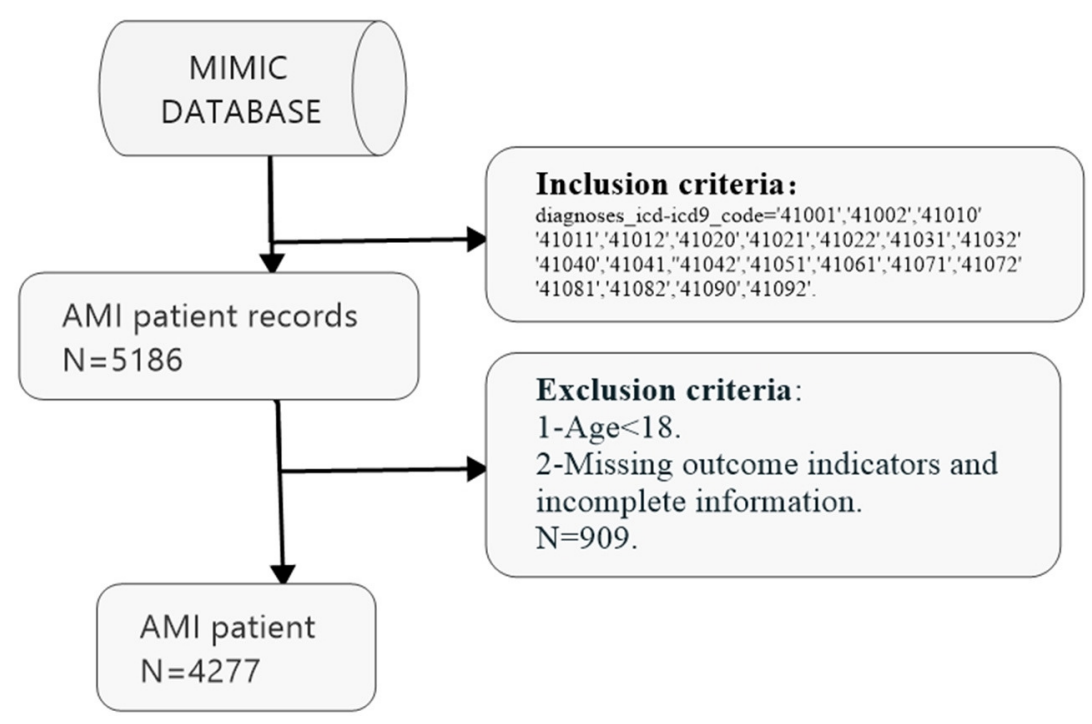

FIGURE 1 | The inclusion and exclusion criteria of this study. 
Assessment (SOFA) score, and Acute Physiology Score (APS)-III when the patient was admitted to the hospital. Survival time (in months) and status were also extracted from the database. The inclusion and exclusion criteria are shown in Figure 1.

\section{Statistical Analysis}

The patients were divided into two groups according to life and death outcomes. In addition to status and survival time,

TABLE 1 | Baseline characteristics of patients in the study.

\begin{tabular}{|c|c|c|c|}
\hline Variable & Live & Dead & $P$-value \\
\hline$N$ & 3,434 & 843 & \\
\hline Sex & & & 0.324 \\
\hline Male & $1,234(35.9)$ & $287(34.0)$ & \\
\hline Female & $2,200(64.1)$ & $556(66.0)$ & \\
\hline AF & & & $<0.001$ \\
\hline Yes & $931(27.1)$ & 323 (38.3) & \\
\hline No & 2,503 (72.9) & $520(61.7)$ & \\
\hline AFL & & & 1 \\
\hline Yes & 79 (2.3) & $20(2.4)$ & \\
\hline No & 3,355 (97.7) & $813(97.6)$ & \\
\hline VF & & & 0.01 \\
\hline Yes & $139(4.0)$ & $52(6.2)$ & \\
\hline No & 3,295 (96.0) & 791 (93.8) & \\
\hline VT & & & $<0.001$ \\
\hline Yes & $281(8.2)$ & $105(12.5)$ & \\
\hline No & 3,153 (91.8) & $738(87.5)$ & \\
\hline Drug & & & 0.001 \\
\hline Yes & $1,829(53.3)$ & 395 (46.9) & \\
\hline No & $1,605(47.7)$ & $448(53.1)$ & \\
\hline Total_Ca & $8.7(8.2-9.1)$ & $8.5(7.9-9.0)$ & $<0.001$ \\
\hline Chloride & 103.0 (100.0-106.0) & $102.0(98.0-106.0)$ & $<0.001$ \\
\hline Creatinine & $1.0(0.8-1.5)$ & $1.5(1.0-2.4)$ & $<0.001$ \\
\hline Phosphate & $3.5(2.9-4.1)$ & $3.9(3.2-5.0)$ & $<0.001$ \\
\hline Potassium & $4.2(3.8-4.6)$ & $4.4(3.9-4.9)$ & $<0.001$ \\
\hline Sodium & $139.0(136.0-141.0)$ & $138.0(135.0-141.0)$ & 0.009 \\
\hline Nitrogen & $21.0(15.0-31.0)$ & $33.0(21.0-51.0)$ & $<0.001$ \\
\hline Hemoglobin & $12.2(10.6-13.7)$ & $11.2(9.9-12.5)$ & $<0.001$ \\
\hline Platelet & 236.0 (188.0-294.0) & 233.0 (173.0-308.5) & 0.347 \\
\hline RDW & $13.8(13.1-14.9)$ & $14.9(13.7-16.7)$ & $<0.001$ \\
\hline WBC & $10.4(8.0-13.7)$ & $12.3(8.9-16.7)$ & $<0.001$ \\
\hline Age & $69.0(58.3-78.0)$ & $77.0(68.0-83.0)$ & $<0.001$ \\
\hline Respiration rate & $25.0(18.5-29.0)$ & $28.0(24.0-33.0)$ & $<0.001$ \\
\hline Mean HR & $80.7(71.2-90.1)$ & $86.2(75.1-97.7)$ & $<0.001$ \\
\hline MBP & $58.0(51.8-83.0)$ & $53.0(46.0-61.0)$ & $<0.001$ \\
\hline DBP & $58.8(53.0-65.7)$ & $55.2(49.5-62.0)$ & $<0.001$ \\
\hline SBP & 113.5 (105.4-124.2) & 108.5 (99.5-122.0) & $<0.001$ \\
\hline Mean glucose & 134.8 (117.2-163.0) & $149.0(121.3-187.1)$ & $<0.001$ \\
\hline SOFA & $3.0(1.0-5.0)$ & $6.0(4.0-9.0)$ & $<0.001$ \\
\hline APS-III & $37.0(28.0-49.0)$ & $55.0(45.0-72.0)$ & $<0.001$ \\
\hline
\end{tabular}

$A F$, atrial fibrillation; $A F L$, atrial flutter; VF, ventricular fibrillation; VT, ventricular tachycardia; $R D W$, red blood cell width; WBC, white blood cell count; Mean HR, mean heart rate; SBP, systolic blood pressure; MBP, mean blood pressure; DBP, diastolic blood pressure; SOFA, Sequential Organ Failure Assessment; APS-III, Acute Physiological Score. the chi-square test was performed on categorical variables, and Student's $t$-test was performed on continuous variables. Then, Cox regression was performed on all variables to explore the variables that have an impact on AMI outcome. Finally, SBP was categorized as low $(<90 \mathrm{mmHg})$, normal (90-140 mmHg), and high (>140 mmHg). A non-linear test was performed to judge whether the effect of SBP on AMI prognosis is non-linear. The patients were also divided into the "advanced age" and "low age" groups for subgroup analysis. Meaningful variables were incorporated in the Cox regression results to construct three models, and then sensitivity analysis was performed on the models. SBP alone was incorporated in model 1; some variables were collectively included in model 2, and all meaningful variables were included in the Cox regression result as model 3. The best cutoff point for the entire prognostic trajectory was determined. $P<0.05$ was considered statistically significant. Statistical analyses were conducted using Excel, SPSS, and R (ggplot2, rms, survival, and segmented packages).

\section{RESULTS}

Among the 4,277 patients with AMI, 843 died during followup. According to our results, gender $(P=0.324)$ may not be a factor affecting AMI outcome. The proportion of male/female (\%) in the survival group was 2,200/1,234 (64.1\%/35.9\%), and that in the death group was 556/287 (66.0\%/34.0\%). The mean value $[95 \%$ confidence interval $(95 \% \mathrm{CI})]$ of the

TABLE 2 | The results of cox regression analysis.

\begin{tabular}{|c|c|c|c|}
\hline Variable & HR & $95 \% \mathrm{Cl}$ & $P$-value \\
\hline Age & 1.0477 & $1.0382-1.0572$ & $<0.001$ \\
\hline VFI & & & $<0.001$ \\
\hline No & Reference & & \\
\hline Have & 1.9324 & $1.3678-2.7302$ & \\
\hline VT & & & $<0.001$ \\
\hline No & Reference & & \\
\hline Have & 1.589 & $1.2232-2.0642$ & \\
\hline Drug & & & 0.027 \\
\hline No & Reference & & \\
\hline Have & 0.8275 & $0.6999-0.9784$ & \\
\hline Total_Ca & 0.8470 & $0.7668-0.9357$ & 0.001 \\
\hline Chloride & 0.9721 & $0.9523-0.9922$ & 0.007 \\
\hline Creatinine & 0.9318 & $0.8817-0.9847$ & 0.012 \\
\hline Phosphate & 1.1209 & $1.0522-1.1940$ & $<0.001$ \\
\hline Platelet & 0.9991 & $0.9984-0.9998$ & 0.011 \\
\hline RDW & 1.1483 & $1.1014-1.1972$ & $<0.001$ \\
\hline WBC & 1.0115 & $1.0043-1.0188$ & 0.002 \\
\hline Respiration rate & 1.0095 & $1.0006-1.0185$ & 0.036 \\
\hline Mean HR & 1.0106 & $1.0044-1.0169$ & $<0.001$ \\
\hline Mean glucose & 1.0035 & $1.0021-1.0049$ & $<0.001$ \\
\hline SOFA & 1.0488 & $1.0106-1.0883$ & 0.012 \\
\hline APS-III & 1.0219 & $1.0162-1.0276$ & $<0.001$ \\
\hline
\end{tabular}


TABLE 3 | Cox regression analyses of the relationship between SBP and AMI prognosis.

\begin{tabular}{|c|c|c|c|c|c|c|}
\hline Variable & \multicolumn{2}{|c|}{ Model 1} & \multicolumn{2}{|c|}{ Model 2} & \multicolumn{2}{|c|}{ Model 3} \\
\hline Low & $6.717(5.044-8.946)$ & $<0.001$ & 4.910 (3.679-6.552) & $<0.001$ & $3.080(2.162-4.385)$ & $<0.001$ \\
\hline High & $1.483(1.120-1.965)$ & 0.01 & $1.637(1.231-2.18)$ & $<0.001$ & $2.937(1.918-4.498)$ & $<0.001$ \\
\hline
\end{tabular}

Model 1: univariate. Model 2: adjust for aged, VF, VT, drug, SOFA, and APS-III. Model 3: adjusted for age, VF, VT, Total_Ca, chloride, creatinine, phosphate, platelet, RDW, WBC, respiration rate, mean HR, mean glucose, drug, SOFA, and APS-III.

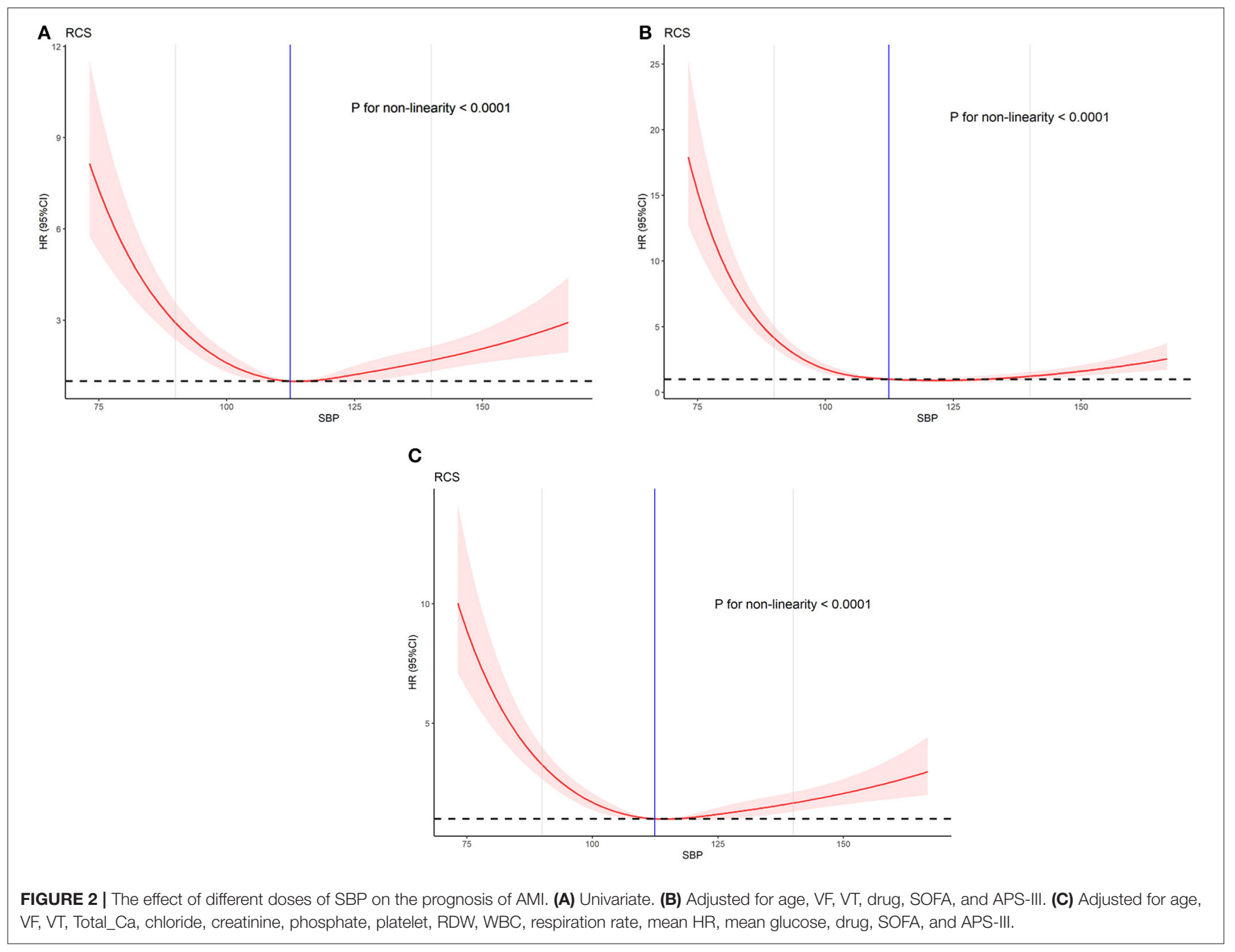

platelet count $(P=0.347)$ of the same patients in the survival group was 236.00 (188.00-294.00), whereas the mean value (95\% CI) in the death group was 233.00 (173.00-308.50). The incidence of AFL in the survival and death groups was $2.3 \%(N=79)$ and $2.4 \%(N=20, P=1)$, respectively. All variables except gender $(P=0.324)$, AFL $(P=1)$, and platelet $(P=0.347)$ showed differences in AMI outcome. The results of the chi-square test and Student's $t$-test are shown in Table 1.
All variables were incorporated into the Cox regression of model 3. The results showed that age, VF, VT, drug, total calcium level, chloride level, creatinine level, phosphate level, platelet count, RDW, WBC count, respiration rate, mean heart rate, mean glucose, SOFA score, and APS-III score are the prognostic factors of AMI (all $P<0.001$, Table 2).

The statistical results of the RCS test are shown in Table 3. Model 1 incorporates a single variable (SBP) into the analysis as shown in Figure 2A. The hazard ratio (HR) with (95\% CI) 
of model 1 with low SBP is 6.717 (5.044-8.946, $P<0.001)$, and that of model 1 with high SBP is $1.483(1.120-1.965, P<$ $0.01)$. Model 2 incorporates age into the analysis as shown in Figure 2B. The HR of model 2 with low SBP is 4.910 (3.6796.552, $P<0.001$ ), and that of model 2 with high SBP is 1.637 (1.231-2.18, $P<0.001)$. Model 3 incorporates all meaningful factors in all Cox regression results into the analysis as shown in Figure 2C. The HR (95\% CI) of model 3 with low SBP is $3.080(2.162-4.385, P<0.001)$, and that of model 2 with high SBP is 2.937 (1.918-4.498). SBP is non-linear in the prognosis curves of AMI in all models. The trend of the curve changed at about SBP $=110 \mathrm{mmHg}$ (Figure 2, all $P<0.001$ ). Notably, the HRs of all models with low SBP are greater than those with high SBP $(P<0.05)$. This finding indicates that the risk of patients with AMI who have SBP $<90 \mathrm{mmHg}$ is higher than that of patients with SBP $>140 \mathrm{mmHg}$. This performance becomes more obvious and statistically significant with the addition of more adjustment variables.

Age is a prognostic factor of AMI in the Cox regression analysis results. Therefore, we divided the patients into the lowage ( $\leq 65$ years) and high-age groups ( $>65$ years) and performed an age subgroup analysis. The baseline characteristics and COX regression analysis results of the subgroups are shown in Table 4 and Table 5. The results of the two groups are all non-linear $(P$ $<0.001$ ). The non-linear curve performance of the two groups is shown in Figure 3. The slope of the low SBP part is steeper than the slope of the high SBP part. This result reflects that the risk of low SBP is higher than that of high SBP in patients with AMI. The results are similar to the performance of all models.

After the prognostic trajectory of SBP was verified as nonlinear, a two-line piecewise linear model with a single change point was estimated by trying all possible values for the change point and choosing the value with the highest likelihood. The result showed that the cutoff point is $114.489 \mathrm{mmHg}(111.275-$ $117.702 \mathrm{mmHg})$. The HR $(95 \% \mathrm{CI})$ of line 1 (SBP $<114.489$ $\mathrm{mmHg})$ is $0.952(0.943-0.962, P<0.001)$. This result means that for every $1 \mathrm{mmHg}$ reduction in SBP, the risk of death in patients with AMI is reduced by $4.8 \%(1-0.952)$. The HR (95\% CI) of line 2 (SBP $>114.489 \mathrm{mmHg}$ ) is $1.026(1.018-1.035, P<0.001)$, which also means that the risk of death in AMI patients increases by $2.6 \%(1.018-1)$ for every $1 \mathrm{mmHg}$ increase in SBP (Figure 4).

\section{DISCUSSION}

In the past few decades, mortality from cardiovascular disease has been greatly reduced (18) partly because of the improved management of AMI (19). However, AMI is the most serious manifestation of CAD and the main cause of global cardiovascular disease morbidity and mortality $(6,20)$. A number of studies have pointed out that SBP is related to the prognostic risk of AMI (11-13, 21, 22). MBP may also be an important predictor. Pulse pressure has a little effect on the risk of cardiovascular disease; thus, SBP should be used in long-term monitoring as an indicator of AMI prognosis (23). Psaty and others pointed out that although DBP, SBP, and pulse pressure are directly related to the risk of coronary and cerebrovascular
TABLE 4 | Baseline characteristics between different age groups.

\begin{tabular}{|c|c|c|c|}
\hline Variable & $\begin{array}{l}\text { Low-age group } \\
\quad(\text { age }<65)\end{array}$ & $\begin{array}{l}\text { High-age group } \\
\quad(\text { age }>65)\end{array}$ & $P$-value \\
\hline$n$ & 1,616 & 2,661 & \\
\hline Status & & & $<0.001$ \\
\hline Dead & $159(9.8)$ & $684(25.7)$ & \\
\hline Live & 1,457 (90.2) & $1,977(74.3)$ & \\
\hline Sex & & & 0.01 \\
\hline Male & $614(38.0)$ & $907(34.1)$ & \\
\hline Female & $1,002(62.0)$ & $1,754(65.9)$ & \\
\hline AFI & & & $<0.001$ \\
\hline Yes & $253(15.7)$ & 1,001 (37.6) & \\
\hline No & 1,363 (84.3) & $1,660(62.4)$ & \\
\hline AFL & & & 0.01 \\
\hline Yes & $24(1.5)$ & $75(2.8)$ & \\
\hline No & 1,592 (98.5) & 2,586 (97.2) & \\
\hline VFI & & & 0.001 \\
\hline Yes & $95(5.9)$ & $96(3.6)$ & \\
\hline No & $1,521(94.1)$ & 2,565 (96.4) & \\
\hline VT & & & 0.01 \\
\hline Yes & 1,445 (89.4) & $215(8.1)$ & \\
\hline No & $171(10.6)$ & 2,446 (91.9) & \\
\hline SBP & & & 0.64 \\
\hline Normal & $1,472(91.1)$ & 2,403 (90.3) & \\
\hline Lower & $38(2.4)$ & $73(2.7)$ & \\
\hline Higher & $106(6.6)$ & $185(7.0)$ & \\
\hline Drug & & & 0.28 \\
\hline Yes & $858(53.1)$ & $1,366(51.3)$ & \\
\hline No & 758 (46.9) & $1,295(48.7)$ & \\
\hline Total_Ca & $8.7(8.1-9.1)$ & $8.6(8.1-9.1)$ & 0.83 \\
\hline Chloride & $103.0(100.0-106.0)$ & $103.0(100.0-106.0)$ & 0.11 \\
\hline Creatinine & $1.0(0.8-1.4)$ & $1.2(0.9-1.7)$ & $<0.001$ \\
\hline Phosphate & $3.5(2.9-4.2)$ & $3.6(3.0-4.3)$ & $<0.001$ \\
\hline Potassium & $4.2(3.8-4.6)$ & $4.2(3.8-4.7)$ & $<0.001$ \\
\hline Sodium & $138.0(136.0-140.0)$ & $138.0(136.0-141.0)$ & 0.06 \\
\hline Nitrogen & $18.0(14.0-27.0)$ & $25.0(18.0-39.0)$ & $<0.001$ \\
\hline Hemoglobin & $12.8(11.0-14.2)$ & $11.6(10.2-13.0)$ & $<0.001$ \\
\hline Platelet & $245.0(196.0-299.0)$ & $230.0(180.0-295.0)$ & $<0.001$ \\
\hline RDW & $13.7(13.0-14.7)$ & $14.2(13.4-15.5)$ & $<0.001$ \\
\hline WBC & $11.0(8.3-14.4)$ & $10.5(8.0-14.2)$ & 0.66 \\
\hline Respiration rate & $25.0(17.5-29.0)$ & $26.0(23.0-30.0)$ & $<0.001$ \\
\hline Mean HR & $82.5(72.6-92.5)$ & $81.2(71.8-91.2)$ & 0.02 \\
\hline Mean BP & $61.0(54.0-87.0)$ & $55.0(49.0-76.2)$ & $<0.001$ \\
\hline Mean glucose & $134.1(117.0-164.3)$ & $139.1(118.5-168.8)$ & 0.01 \\
\hline SOFA & $3.0(1.0-5.3)$ & $4.0(2.0-7.0)$ & $<0.001$ \\
\hline APS-III & $34.0(25.0-48.0)$ & $44.0(34.0-57.0)$ & $<0.001$ \\
\hline
\end{tabular}

events, SBP is the best single predictor of cardiovascular events (22).

The results of our Cox multivariate analysis study showed that SBP $(P<0.001)$ is an influencing factor for AMI prognosis. All the prognostic curves of SBP in the RCS shows very similar performance. Our study sets a normal value range (90-140 
TABLE 5 | Cox regression results for different age groups.

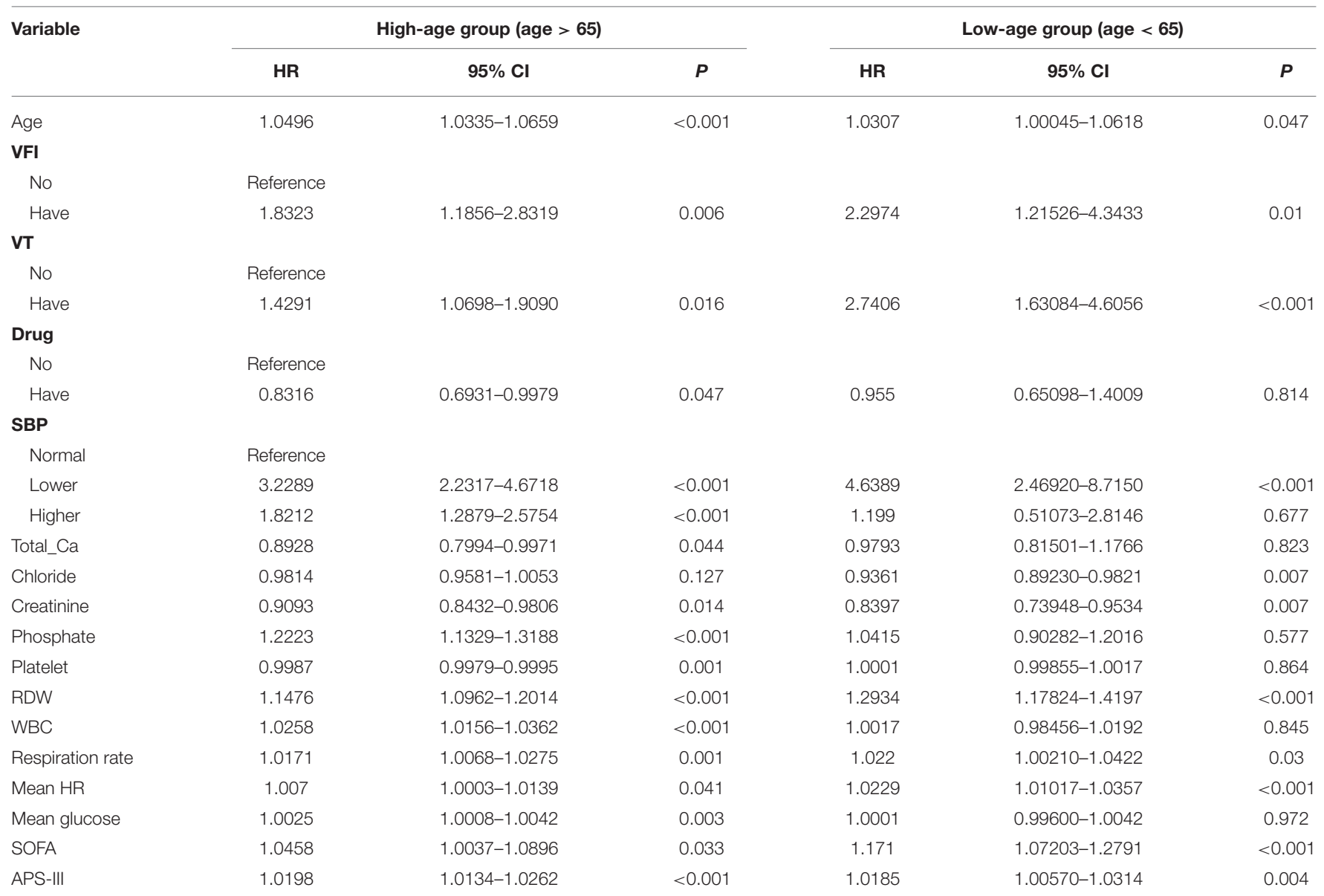

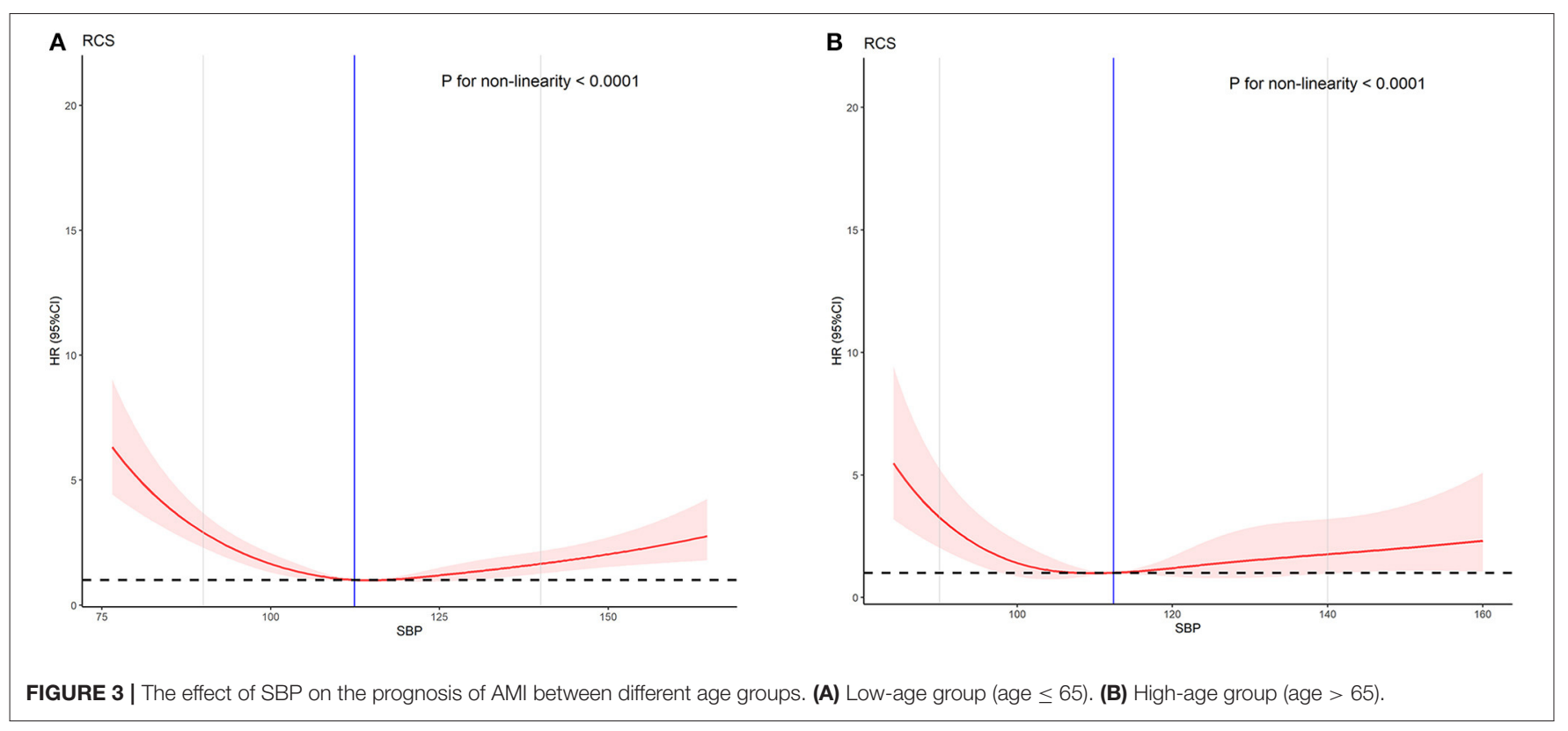

$\mathrm{mmHg}$ ) based on the normal physiological values in the human body, and the risk of AMI is the lowest within the normal value range. Notably, the performance of SBP in the prognosis of AMI in the RCS analysis is non-linear, and the non-linear test is statistically significant $(P<0.001)$. Moreover, in the sensitivity analysis, all models with low SBP had higher risks than those with 
high SBP. The risks in models 1-3 with low SBP are 6.717, 4.910, and 3.080 times those with normal SBP, and the risks in models 1-3 with high SBP are 1.483, 1.637, and 2.937 times those with normal SBP, respectively. In the subgroup analysis, the low-age and high-age groups also showed a higher risk among patients with low SBP compared with those with high SBP.

When the SBP of a patient with AMI is low to a certain level, the blood supply to the coronary artery becomes obstructed, which leads to myocardial ischemia, myocardial hypoxia, degeneration and necrosis, and eventually myocardial infarction. Several observational studies have shown that lowering the BP below a certain threshold may be harmful as reflected by the " $\mathrm{J}$ curve phenomenon" (24-26). An observational study of 22,672 patients with stable CAD showed that hypertension, low SBP $(<120 \mathrm{mmHg})$, and low DBP $(<70 \mathrm{mmHg})$ are all related to the heart. The increased risk of vascular events supported the "J curve phenomenon"; thus, low BP may be harmful in patients with coronary heart disease (27). Shiraishi et al. found that patients with AMI who have SBP $<106 \mathrm{mmHg}$ often reach Killip $\geq 3$ upon hospital admission. The right coronary artery and left main trunk or multivessels are the culprits, the number of diseased blood vessels increases, and the hospital mortality rate increased considerably (28). The results of the Israeli Acute Coronary Syndrome Survey showed that SBP is related to cardiovascular events and total mortality. Patients with SBP $<110 \mathrm{mmHg}$ have a remarkably higher 1-year mortality risk ratio within 7 days (HR $=2.37$ ) compared with those with normal SBP $(110-140 \mathrm{mmHg}$, HR = 1.92) upon admission (29). Our study determined that the cutoff value of the SBP prognostic curve is $114.489 \mathrm{mmHg}$, and we quantified the risk of the entire curve. The result means that for every $1 \mathrm{mmHg}$ reduction in SBP before $114.489 \mathrm{mmHg}$, the risk of death in patients with AMI is reduced by $4.8 \%$ (10.952). Moreover, the risk of death among patients with AMI increases by $2.6 \%(1.018-1)$ for every $1 \mathrm{mmHg}$ increase in SBP after $114.489 \mathrm{mmHg}$. Lewington et al. also pointed out that SBP = $115 \mathrm{mmHg}$ is an important observation threshold $(30,31)$. This value is very close to our findings.

SBP can reflect cardiac output and systemic peripheral resistance. A higher SBP at admission may indicate increased systemic resistance, maintenance of cardiac function, and less myocardial damage in patients with AMI. Metabolic syndrome refers to the co-occurrence of several known cardiovascular risk factors, including insulin resistance, obesity, atherogenic dyslipidemia, and hypertension. These conditions are interrelated and share underlying mediators, mechanisms, and pathways (32). Abnormal metabolism and blood pressure characterized by metabolic syndrome are risk factors for cardiovascular disease (33), particularly in young patients with AMI. These patients present extensive atherosclerotic disease in angiographic studies (34). Average arterial BP levels and shortterm BP variability are related to hypertension-mediated organ damage, increased carotid intima-media thickness (35), and hypoperfusion, which further increase the risk of adverse events in AMI. The short-term outcomes of patients with normal SBP and with high admission SBP are similar, but with the passage of time, the outcome of patients with excessively elevated SBP upon admission is death, and the probability of major adverse

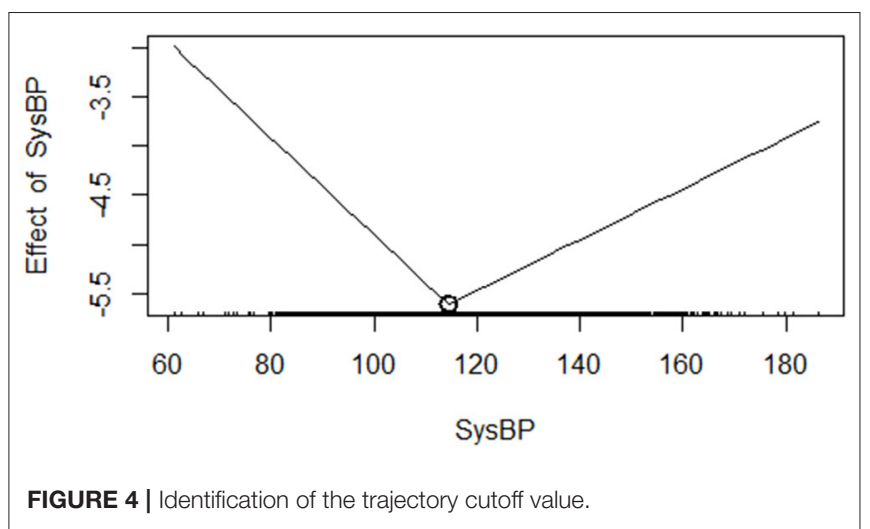

cardiac events has an upward trend (9). Although different studies set different standards for normal BP, the results obtained are consistent, that is, patients with low SBP have a higher risk compared with patients with high SBP. Future studies would be interesting to explore the optimal blood pressure cutpoint by other methods, such as ambulatory blood pressure monitoring or home blood pressure measurement $(36,37)$.

\section{CONCLUSION}

The risk of patients with AMI who have low SBP is very high; therefore, maintaining a high degree of vigilance for patients with AMI and low SBP is necessary. Continuous BP monitoring is particularly important; a normal SBP can guarantee a good prognosis for AMI, and high SBP has a certain low risk. The SBP value of $114.489 \mathrm{mmHg}$ is the inflection point of the prognostic trajectory of AMI. Patients with AMI have the lowest probability of death when the SBP value is $114.489 \mathrm{mmHg}$. Continuous in-depth research on SBP is very necessary. SBP has a nonlinear performance in AMI prognosis. Thus, the establishment of an accurate and reliable prognostic model that uses SBP to predict AMI has still a long way to go. We will further study the impact of SBP on AMI and strive to establish a prediction model with accurate threshold, high sensitivity, and reliability to guide clinicians and assist in decision-making.

\section{LIMITATIONS}

First, our data were obtained from the MIMIC database. Most of the patients included in this database are from the United States; therefore, the generalization of the conclusion is limited. Second, parts of the index information of patients were not fully recorded, which led to the loss of information in the study. Finally, this study is a retrospective study, which may inevitably have some biases.

\section{DATA AVAILABILITY STATEMENT}

The original contributions presented in the study are included in the article/supplementary material, further inquiries can be directed to the corresponding author. 


\section{AUTHOR CONTRIBUTIONS}

SZ: resources, investigation, validation, writing-original draft, and writing-review and editing. FZ: resources, investigation, validation, and writing-review and editing. RY: resources, investigation, and writing-review and editing. WW: resources, investigation, and formal analysis. HL, WM, FX, and $\mathrm{DH}$ : data curation and formal analysis. JL: project administration,

\section{REFERENCES}

1. Moran AE, Forouzanfar MH, Roth GA, Mensah GA, Ezzati M, Flaxman A, et al. The global burden of ischemic heart disease in 1990 and 2010: the Global Burden of Disease 2010 study. Circulation. (2014) 129:1493501. doi: 10.1161/CIRCULATIONAHA.113.004046

2. Mozaffarian D, Benjamin EJ, Go AS, Arnett DK, Blaha MJ, Cushman $\mathrm{M}$, et al. Heart disease and stroke statistics-2015 update: a report from the American Heart Association. Circulation. (2015) 131:e29322. doi: 10.1161/CIR.0000000000000152

3. Nichols M, Townsend N, Scarborough P, Rayner M. Cardiovascular disease in Europe 2014: epidemiological update. Eur Heart J. (2014) 35:2929. doi: 10.1093/eurheartj/ehu299

4. Fox KA, Steg PG, Eagle KA, Goodman SG, Anderson FA, Jr., Granger CB, et al. Decline in rates of death and heart failure in acute coronary syndromes, 1999-2006. Jama. (2007) 297:1892-900. doi: 10.1001/jama.297.17.1892

5. White HD, Chew DP. Acute myocardial infarction. Lancet. (2008) 372:57084. doi: 10.1016/S0140-6736(08)61237-4

6. Reed GW, Rossi JE, Cannon CP. Acute myocardial infarction. Lancet. (2017) 389:197-210. doi: 10.1016/S0140-6736(16)30677-8

7. Townsend N, Nichols M, Scarborough P, Rayner M. Cardiovascular disease in Europe-epidemiological update 2015. Eur Heart J. (2015) 36:2696705. doi: 10.1093/eurheartj/ehv428

8. Wang JC, Normand SL, Mauri L, Kuntz RE. Coronary artery spatial distribution of acute myocardial infarction occlusions. Circulation. (2004) 110:278-84. doi: 10.1161/01.CIR.0000135468.67850.F4

9. Huang B, Yang Y, Zhu J, Liang Y, Tan H. Clinical characteristics and shortterm outcomes in patients with elevated admission systolic blood pressure after acute ST-elevation myocardial infarction: a population-based study. BMJ Open. (2014) 4:e005097. doi: 10.1136/bmjopen-2014-005097

10. Cohn JN. Neuroendocrine activation after acute myocardial infarction. Am J Cardiol. (1990) 65:28i-31i. doi: 10.1016/0002-9149(90)9 0122-H

11. Sheps SG, Black HR, Cohen JD, Kaplan NM, Ferdinand KC, Chobanian AV. The sixth report of the Joint National Committee on prevention, detection, evaluation, and treatment of high blood pressure. Arch Intern Med. (1997) 157:2413-46. doi: 10.1001/archinte.157.21.2413

12. Sesso HD, Stampfer MJ, Rosner B, Hennekens CH, Gaziano JM, Manson JE, et al. Systolic and diastolic blood pressure, pulse pressure, and mean arterial pressure as predictors of cardiovascular disease risk in Men. Hypertension. (2000) 36:801-7. doi: 10.1161/01.HYP.36.5.801

13. Sundström J, Arima H, Jackson R, Turnbull F, Rahimi K, Chalmers $\mathrm{J}$, et al. Effects of blood pressure reduction in mild hypertension: a systematic review and meta-analysis. Ann Intern Med. (2015) 162:18491. doi: 10.7326/M14-0773

14. Pei J, Wang X, Xing Z, Chen P, Su W, Deng S, et al. Association between admission systolic blood pressure and major adverse cardiovascular events in patients with acute myocardial infarction. PLoS ONE. (2020) 15:e0234935. doi: 10.1371/journal.pone. 0234935

15. Yang J, Li Y, Liu Q, Li L, Feng A, Wang T, et al. Brief introduction of medical database and data mining technology in big data era. J Evid Based Med. (2020) 13:57-69. doi: 10.1111/jebm.12373

16. Johnson AEW, Pollard TJ, Shen L, Lehman LWH, Feng M, Ghassemi M, et al. MIMIC-III, a freely accessible critical care database. Sci Data. (2016) 3:160035. doi: $10.1038 /$ sdata.2016.35 supervision, and visualization. All authors contributed to the article and approved the submitted version.

\section{ACKNOWLEDGMENTS}

The authors would like to thank the MIMIC program for providing open access to the database.

17. Saeed M, Villarroel M, Reisner AT, Clifford G, Lehman LW, Moody $\mathrm{G}$, et al. Multiparameter Intelligent Monitoring in Intensive Care II: a public-access intensive care unit database. Crit Care Med. (2011) 39:95260. doi: 10.1097/CCM.0b013e31820a92c6

18. Mozaffarian D, Benjamin EJ, Go AS, Arnett DK, Blaha MJ, Cushman $\mathrm{M}$, et al. Heart disease and stroke statistics-2016 update: a report from the American Heart Association. Circulation. (2016) 133:e38360. doi: 10.1161/CIR.0000000000000350

19. Ford ES, Ajani UA, Croft JB, Critchley JA, Labarthe DR, Kottke TE, et al. Explaining the decrease in U.S. deaths from coronary disease, 1980-2000. N Engl J Med. (2007) 356:2388-98. doi: 10.1056/NEJMsa053935

20. Heusch G, Gersh BJ. The pathophysiology of acute myocardial infarction and strategies of protection beyond reperfusion: a continual challenge. Eur Heart J. (2017) 38:774-84. doi: 10.1093/eurheartj/ehw224

21. Ma WF, Liang Y, Zhu J, Yang YM, Tan HQ Yu LT, et al. Comparison of 4 admission blood pressure indexes for predicting 30-Day mortality in patients with ST-segment elevation myocardial infarction. Am J Hypertens. (2016) 29:332-9. doi: 10.1093/ajh/hpv109

22. Psaty BM, Furberg CD, Kuller LH, Cushman M, Savage PJ, Levine D, et al. Association between blood pressure level and the risk of myocardial infarction, stroke, and total mortality: the cardiovascular health study. Arch Intern Med. (2001) 161:1183-92. doi: 10.1001/archinte.161.9.1183

23. Miura K, Nakagawa H, Ohashi Y, Harada A, Taguri M, Kushiro T, et al. Four blood pressure indexes and the risk of stroke and myocardial infarction in Japanese men and women: a meta-analysis of 16 cohort studies. Circulation. (2009) 119:1892-8. doi: 10.1161/CIRCULATIONAHA.108.823112

24. Bangalore S, Messerli FH, Wun CC, Zuckerman AL, DeMicco D, Kostis JB, et al. J-curve revisited: an analysis of blood pressure and cardiovascular events in the Treating to New Targets (TNT) Trial. Eur Heart J. (2010) 31:2897-908. doi: 10.1093/eurheartj/ehq328

25. Cooper-DeHoff RM, Gong Y, Handberg EM, Bavry AA, Denardo SJ, Bakris GL, et al. Tight blood pressure control and cardiovascular outcomes among hypertensive patients with diabetes and coronary artery disease. JAMA. (2010) 304:61-8. doi: 10.1001/jama.2010.884

26. Mancia G, Schumacher H, Redon J, Verdecchia P, Schmieder $\mathrm{R}$, Jennings $\mathrm{G}$, et al. Blood pressure targets recommended by guidelines and incidence of cardiovascular and renal events in the Ongoing Telmisartan Alone and in Combination With Ramipril Global Endpoint Trial (ONTARGET). Circulation. (2011) 124:1727-36. doi: 10.1161/CIRCULATIONAHA.110.008870

27. Vidal-Petiot E, Ford I, Greenlaw N, Ferrari R, Fox KM, Tardif JC, et al. Cardiovascular event rates and mortality according to achieved systolic and diastolic blood pressure in patients with stable coronary artery disease: an international cohort study. Lancet. (2016) 388:214252. doi: 10.1016/S0140-6736(16)31326-5

28. Shiraishi J, Kohno Y, Sawada T, Ito D, Kimura M, Ariyoshi M, et al. Systolic blood pressure at admission, clinical manifestations, and in-hospital outcomes in patients with acute myocardial infarction. J Cardiol. (2011) 58:54-60. doi: 10.1016/j.jjcc.2011.04.003

29. Shlomai G, Kopel E, Goldenberg I, Grossman E. The association between elevated admission systolic blood pressure in patients with acute coronary syndrome and favorable early and late outcomes. J Am Soc Hypertens. (2015) 9:97-103. doi: 10.1016/j.jash.2014.11.005

30. Lewington S, Clarke R, Qizilbash N, Peto R, Collins R, Prospective Studies Collaboration. Age-specific relevance of usual blood pressure to vascular mortality: a meta-analysis of individual data for one million adults 
in 61 prospective studies. Lancet (London, England). (2002) 360:190313. doi: 10.1016/S0140-6736(02)11911-8

31. Kalkman DN, Brouwer TF, Vehmeijer JT, Berger WR, Berger WR, de Winter RJ, et al. J Curve in patients randomly assigned to different systolic blood pressure targets: an experimental approach to an observational paradigm. Circulation. (2017) 136:2220-9. doi: 10.1161/CIRCULATIONAHA.117.030342

32. Huang PL. A comprehensive definition for metabolic syndrome. Dis Models Mech. (2009) 2:231-7. doi: 10.1242/dmm.001180

33. Cuspidi C, Sala C, Zanchetti A. Metabolic syndrome and target organ damage: role of blood pressure. Expert Rev Cardiovasc Ther. (2008) 6:73143. doi: 10.1586/14779072.6.5.731

34. Ranjith N, Pegoraro RJ, Naidoo DP, Esterhuizen TM. Metabolic syndrome in young Asian Indian patients with myocardial infarction. Cardiovasc J Africa. (2007) 18:228-33.

35. Tatasciore A, Di Nicola M, Tommasi R, Santarelli F, Palombo C, Parati G. From short-term blood pressure variability to atherosclerosis: relative roles of vascular stiffness and endothelial dysfunction. J Clin Hypertens (Greenwich, Conn). (2020) 22:1218-27. doi: 10.1111/jch.13871

36. Zhu H, Liang X, Pan XF, Huang C, Kuang J, Lv W, et al. prospective cohort study of home blood pressure monitoring based on an intelligent cloud platform (the HBPM-iCloud study): rationale and design. Ther Adv Chronic Dis. (2020) 11:2040622320933108. doi: 10.1177/2040622320933108
37. Zhu $\mathrm{H}$, Zheng $\mathrm{H}$, Liu $\mathrm{X}$, Mai W, Huang $\mathrm{Y}$. Clinical applications for out-of-office blood pressure monitoring. Ther Adv Chronic Dis. (2020) 11:2040622320901660. doi: 10.1177/2040622320 901660

Conflict of Interest: The authors declare that the research was conducted in the absence of any commercial or financial relationships that could be construed as a potential conflict of interest.

Publisher's Note: All claims expressed in this article are solely those of the authors and do not necessarily represent those of their affiliated organizations, or those of the publisher, the editors and the reviewers. Any product that may be evaluated in this article, or claim that may be made by its manufacturer, is not guaranteed or endorsed by the publisher.

Copyright (c) 2021 Zheng, Zhao, Yang, Wu, Liu, Ma, Xu, Han and Lyu. This is an open-access article distributed under the terms of the Creative Commons Attribution License (CC BY). The use, distribution or reproduction in other forums is permitted, provided the original author(s) and the copyright owner(s) are credited and that the original publication in this journal is cited, in accordance with accepted academic practice. No use, distribution or reproduction is permitted which does not comply with these terms. 